\title{
Interleukin-17 induces angiogenesis in vitro via CXCL8 and CCL2 in retinal pigment epithelium
}

\author{
YING CHEN $^{1 *}$, MURUI ZHONG ${ }^{2 *}$, GANGXIANG YUAN $^{1}$ and HUI PENG $^{1}$ \\ ${ }^{1}$ Department of Ophthalmology, First Affiliated Hospital of Chongqing Medical University, \\ Chongqing Key Laboratory of Ophthalmology, Chongqing Eye Institute, Chongqing 400016; \\ ${ }^{2}$ Department of Ophthalmology, 452 Hospital of People's Liberation Army, Chengdu, Sichuan 610065, P.R. China
}

Received June 6, 2017; Accepted November 30, 2017

DOI: $10.3892 / \mathrm{mmr} .2018 .8460$

\begin{abstract}
Interleukin-17 (IL-17) is a major pro-inflammatory cytokine involved in choroidal endothelial cell (CEC) angiogenesis. Proteins expressed by the retinal pigment epithelium (RPE) may contribute to CEC angiogenesis. The ability of IL-17 to promote proliferation, migration and capillary-like structure formation in CECs was investigated by stimulating the RPE in vitro. CECs were cultured in a conditioned medium (CM) with IL-17 (IL-17-CM) or without IL-17 (CM) obtained from the supernatant of an ARPE-19 cell line. The pro-angiogenic role of IL-17-CM on CECs was investigated with water-soluble tetrazolium 1 analysis, wound healing and Matrigel matrix tube formation assays. The expression level of vascular endothelial growth factor was detected by enzyme-linked immunosorbent assay in RPE cells treated with or without IL-17. Ras-related C3botulinum toxin substrate 1 (Rac1) and Ras homolog gene family member A (RhoA) activities were analyzed by pull-down assays. IL-17-CM significantly enhanced tube formation and increased the migration distance in CECs in comparison with CM. This effect was diminished by neutralizing C-C motif chemokine 2 (CCL2) and $\mathrm{C}-\mathrm{X}-\mathrm{C}$ motif chemokine ligand 8 (CXCL8) expression in IL-17-CM, with a concomitant downregulation of Rac1 and RhoA activity in CECs. In conclusion, it was demonstrated that IL-17 mediated the expression of CCL2 and CXCL8 in RPE cells, resulting in increased migration and tube formation in human CECs.
\end{abstract}

Correspondence to: Dr Hui Peng, Department of Ophthalmology, First Affiliated Hospital of Chongqing Medical University, Chongqing Key Laboratory of Ophthalmology, Chongqing Eye Institute, 1 You Yi Road, Yu Zhong, Chongqing 400016, P.R. China E-mail: penghui20140614@126.com

${ }^{*}$ Contributed equally

Key words: retinal pigment epithelium, choroidal endothelial cell, neovascularization, $\mathrm{C}-\mathrm{C}$ motif chemokine ligand $2, \mathrm{C}-\mathrm{X}-\mathrm{C}$ motif chemokine ligand 8

\section{Introduction}

Age-related macular degeneration (AMD) is the leading cause of irreversible visual impairment in elderly populations worldwide (1). It may be classed as wet or dry, with wet AMD accounting for $\sim 90 \%$ of the severe vision loss associated with AMD, despite only affecting $\sim 10 \%$ of individuals with the disease.

Wet AMD is associated with choroidal neovascularization (CNV) (1), which is characterized by the proliferation of blood vessels that originates in the choroid and grow through the Bruch's membrane and into the sub-retinal space. These blood vessels eventually bleed and/or leak fluid, leading to central vision loss (2). The proliferation, migration and capillary-like structure formation of choroidal endothelial cells (CECs) has critical involvement in CNV development (3). The mechanisms underlying $\mathrm{CNV}$ development beneath the neurosensory retina in AMD are complex and have not been fully elucidated.

In wet AMD, the retinal pigment epithelium (RPE) may separate from the Bruch's membrane and become dysfunctional (4,5). Physiologically, RPE cells maintain the structural integrity of the retina and choriocapillaris by secreting numerous growth factors and nutrients essential to the survival of the photoreceptors. The alteration in RPE growth factor secretion contributes to CNV development (4,6-8). In neovascularized tissues obtained from patients with AMD, the RPE secretes vascular endothelial growth factor (VEGF) at an increased rate in comparison to tissues with no neovascularization (9). Furthermore, the RPE may be induced to secrete inflammatory chemokines including $\mathrm{C}-\mathrm{C}$ motif chemokine 2 (CCL2, also termed monocyte chemotactic protein 1) and C-X-C motif chemokine ligand 8 (CXCL8, also termedinterleukin-8) by numerous stimuli, which have been demonstrated to promote neovascularization in vitro $(10,11)$.

Interleukin-17 (IL-17) is primarily produced by cluster of differentiation CD4+ $\mathrm{T}$ cells and is a major pro-inflammatory cytokine associated with the pathogenesis of various autoimmune and inflammatory diseases $(12,13)$. IL-17 is detected at high levels in the serum of patients with AMD (14). In a laser-induced choroidal neovascularization rat model, IL-17A mRNA expression is increased in the retina (15). A previous study demonstrated that IL-17 induces RPE to secrete CCL2 and CXCL8 (16), exhibiting direct angiogenic activity via 
activation of the phosphoinositide 3-kinase-Ras-related C3 botulinum toxin substrate 1 (Racl) and Ras homolog gene family member A (RhoA) pathways in CECs in vitro (17). However, it is still unclear if IL-17 affects CECs through RPE activation.

In the present study, the supernatant of the RPE was stimulated by IL-17 to obtain an IL-17-conditioned medium (IL-17-CM). Its effects on CEC proliferation, migration and tube formation were investigated in a human CEC model.

\section{Materials and methods}

Cell culture. Informed consent was obtained from the relatives of all donors. The study was conducted in accordance with The Declaration of Helsinki and was approved by the institutional review board of The First Affiliated Hospital of Chongqing Medical University (Chongqing, China). Human ocular posterior segments free of any known ocular disease were obtained from Chongqing Eye Bank (Chongqing, China). The retina was torn from RPE with forceps, and the RPE was rubbed off of the choroid with a cotton swab. Choroids were torn from sclera, and endothelial cells were isolated from 2 choroids of 1 donor by treatment with $0.2 \%$ type II collagenase in minimum essential medium (Gibco; Thermo Fisher Scientific, Inc., Waltham, MA, USA) at $37^{\circ} \mathrm{C}$ and $5 \% \mathrm{CO}_{2}$ until the tissue was visibly digested. The cell suspension was filtered through sterile 70 and 40-mm filters (BD Biosciences, Franklin Lakes, NJ, USA). The filtered suspension was centrifuged at $300 \mathrm{x} \mathrm{g}$ and suspended in medium containing anti-CD31 magnetic beads (MiltenyiBiotec, $\mathrm{GmbH}$, Bergisch Gladbach, Germany) according to the manufacturer's protocol. CECs were isolated from human choroid and cultured in endothelial growth medium (EGM-2 MV; Lonza Group Ltd., Basel, Switzerland) supplemented with 5\% fetal bovine serum (FBS; Invitrogen; Thermo Fisher Scientific, Inc.) at $37^{\circ} \mathrm{C}$ in a humidified atmosphere with $5 \% \mathrm{CO}_{2}$, as previously described (17). CECs between passages three and five were used for experiments. ARPE-19 cells were obtained from the American Type Culture Collection and maintained in Dulbecco's modified Eagle's medium/F12 (DMEM/F12; 1:1; Invitrogen; Thermo Fisher Scientific, Inc.) supplemented with 10\% FBS (Invitrogen; Thermo Fisher Scientific, Inc.), $100 \mathrm{U} / \mathrm{ml}$ penicillin and $100 \mu \mathrm{g} / \mathrm{ml}$ streptomycin in a humidified incubator at $37^{\circ} \mathrm{C}$ in $5 \% \mathrm{CO}_{2}$.

ARPE-19 monolayer cultures were seeded at $2 \times 10^{5} \mathrm{cells} / \mathrm{cm}^{2}$ onto Transwell filters (diameter, $12 \mathrm{~mm}$; pore size, $0.4 \mu \mathrm{m}$ ) which had been coated with $160 \mathrm{ml}$ Matrigel (dilution, 1:30; BD Biosciences, Franklin Lakes, NJ) in DMEM/F12 (Invitrogen; Thermo Fisher Scientific, Inc.) and air-dried overnight, as previously described (18). DMEM/F12 (Invitrogen; Thermo Fisher Scientific, Inc.) medium supplemented with $0.5 \%$ FBS (Invitrogen; Thermo Fisher Scientific, Inc.) was used to culture the ARPE-19 cells. The medium was replaced every 4 days and cells were maintained for 8 weeks.

The ARPE-19 monolayer was subsequently cultured with or without $20 \mathrm{ng} / \mathrm{ml} \mathrm{IL-17} \mathrm{(R \& D} \mathrm{Systems,} \mathrm{Inc.,} \mathrm{Minneapolis,}$ MN, USA) in EGM-2 MV medium (Lonza Group Ltd.) for $48 \mathrm{~h}$, and conditioned medium with $20 \mathrm{ng} / \mathrm{ml} \mathrm{IL-17}$ (IL-17-CM) or without IL-17(CM) was obtained from the lower chamber of the ARPE-19 monolayer. To avoid the stimulation of
CECs by redundant IL-17 in IL-17-CM and to reasonably match the control conditions, both CM and IL-17-CM were supplemented with $0.2 \mathrm{ug} / \mathrm{ml}$ neutralizing antibodies (cat. no. AF-317-NA; R\&D Systems, Inc.) against IL-17for $10 \mathrm{~min}$, at room temperature, prior to use.

Cell proliferation assay. CECs were seeded at $2 \times 10^{3}$ cells per well in 96-well plates (Corning Incorporated, Corning, NY, USA) and starved in FBS free EGM-2 MV medium (Lonza Group Ltd) for $12 \mathrm{~h}$. Cells were subsequently treated with CM or IL-17-CM for 24, 48 or $72 \mathrm{~h}$. Cell proliferation was measured according to the Cell Proliferation Reagent Water Soluble Tetrazolium 1 (WST-1) kit manufacturer's protocol (C0035; Beyotime Institute of Biotechnology, Shanghai, China). Cells were incubated in WST-1 solution for $2 \mathrm{~h}$ at $37^{\circ} \mathrm{C}$ and the absorbance at a wavelength of $450 \mathrm{~nm}$ was measured using a spectrophotometer (SpectraMax; Molecular Devices, LLC, Sunnyvale, CA, USA). All measurements were taken in quadruplicate.

Cell migration assay. CECs were cultured to confluence in 6-well plates containing EGM-2 MV (Lonza Group Ltd) supplemented with 5\% FBS (Invitrogen; Thermo Fisher Scientific, Inc.) and subsequently starved in FBS free EGM-2 $\mathrm{MV}$ for $12 \mathrm{~h}$. The monolayer was injured with a sterile pipette tip and washed with PBS to remove debris. The injured monolayer was treated with CM, IL-17-CM or IL-17-CM with $0.1 \mu \mathrm{g} / \mathrm{ml}$ neutralizing antibody against CXCL8 (R\&D Systems, Inc., cat. no. AF-208-NA) and $/$ or $0.2 \mu \mathrm{g} / \mathrm{ml}$ neutralizing antibody against CCL2 (R\&D Systems, Inc. cat. no. AF-279-NA), for $10 \mathrm{~min}$ at room temperature. Images were taken at $0,4,8$ and $12 \mathrm{~h}$ with a fluorescence microscope (DM6000; Leica, Wentzler, Germany). Wound closure rate was measured using Image Pro Plus 6.0 software (Media Cybernetics, Inc., Rockville, MD, USA) and measurements were taken in triplicate.

Tube formation assay. Ice-cold growth factor reduced basement membrane matrixgel (Matrigel; BD Biosciences) was added at $300 \mu \mathrm{l}$ per well to pre-cooled 24 -well plates at $4^{\circ} \mathrm{C}$ and allowed to polymerize at $37^{\circ} \mathrm{C}$ for $30 \mathrm{~min}$. CECs $\left(1 \times 10^{5}\right)$ were plated onto the gel surface and incubated at $37^{\circ} \mathrm{C}$ for $16 \mathrm{~h}$ with $200 \mu \mathrm{l} \mathrm{CM}$, IL-17-CM or IL-17-CM with anti-CXCL8 and/or anti-CCL2 antibodies. Cell rearrangement and tube formation was visualized using phase-contrast microscopy. Images of five random fields at $\mathrm{x} 5$ magnification per well were taken and endothelial tube length was quantified using Image Pro Plus 6.0 software (Media Cybernetics, Inc.).

Enzyme-linked immunosorbent assay (ELISA). The ARPE-19 monolayer was maintained in DMEM/F12 (Invitrogen; Thermo Fisher Scientific, Inc.) medium supplemented with 0.5\% FBS (Invitrogen; Thermo Fisher Scientific, Inc.) for 8 weeks to allow polarization. The cells were then treated with or without IL-17 $(50 \mathrm{ng} / \mathrm{ml})$ for $24 \mathrm{~h}$. The supernatant of the lower chamber was centrifuged at $300 \mathrm{x} \mathrm{g}, 37^{\circ} \mathrm{C}$ for $5 \mathrm{~min}$ to remove particulates and stored at $-70^{\circ} \mathrm{C}$ until further use. Total protein for each sample was determined using a protein assay kit (Bio-Rad Laboratories, Inc. Hercules, CA, USA). VEGF levels were measured using a human ELISA development kit (R\&D Systems, Inc. cat. no. SVE00) according to the 
A

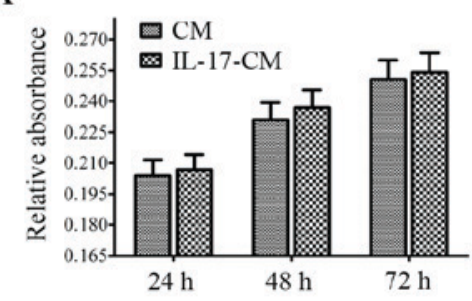

B

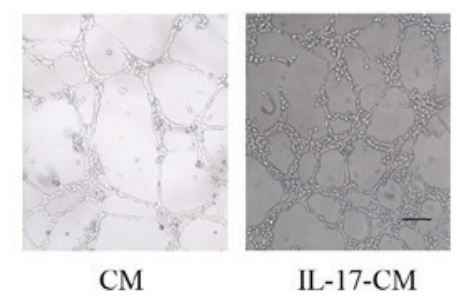

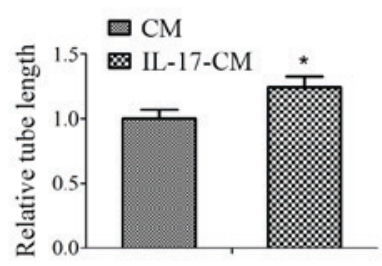

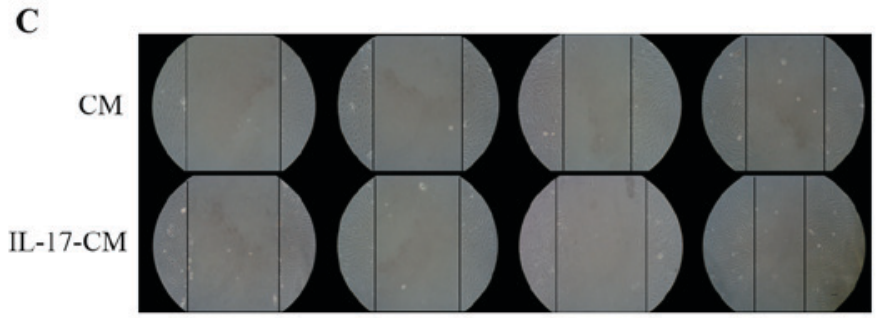

$0 \mathrm{~h}$
$4 \mathrm{~h}$
$8 \mathrm{~h}$

$12 \mathrm{~h}$

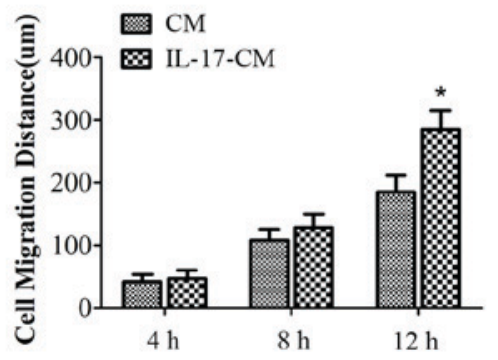

Figure 1. Effect of IL-17-CM on CEC proliferation, migration and tube formation. (A) Cell proliferation was measured with a water soluble tetrazolium 1 kit at 24, 48 and $72 \mathrm{~h}$. (B) Cells were seeded onto Matrigel-coated plates and exposed to CM or IL-17-CM for $16 \mathrm{~h}$. Endothelial tube formation was visualized by microscopy and the tube length was analyzed. Scale bar, $100 \mu \mathrm{m}$. Relative absorbance and tube length data was normalized to a CM value of 1 . (C) CEC monolayers were scratched and incubated with CM or IL-17-CM. Wound healing distance was recorded by phase contrast microscopy at $0,4,8$ and $12 \mathrm{~h}$ following the scratch injury. Scale bar, $100 \mu \mathrm{m}$. Data are presented as the mean \pm standard deviation of four replicate experiments in A, five in B and three in C. ${ }^{*} \mathrm{P}<0.05$ vs. the CM. IL-17, interleukin-17; CM, conditioned medium; CEC, choroidal endothelial cell.

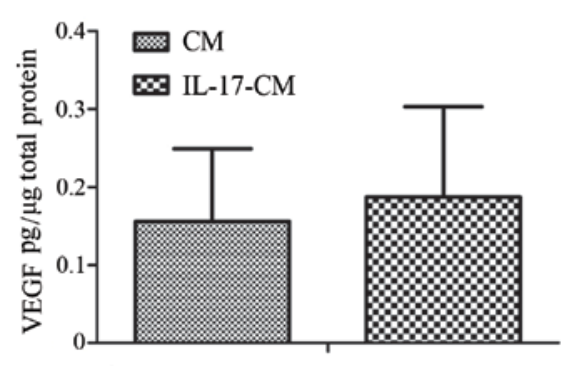

Figure 2. VEGF expression levels in CM and IL-17-CM (pg/ $/ \mu \mathrm{g}$ total protein). Confluent ARPE-19 cells were stimulated with or without IL-17 $(20 \mathrm{ng} / \mathrm{ml})$ for $24 \mathrm{~h}$. The level of VEGF released into the supernatant was quantified by ELISA. Data is presented as the mean \pm standard deviation of four independent experiments. VEGF, vascular endothelial growth factor; $\mathrm{CM}$, conditioned medium; IL-17, interleukin-17.

manufacturer's protocol. Each experiment was repeated four times.

Racl and RhoA pull-down assay. Rac1 and RhoA activity was analyzed using Rac1 and RhoA pull-down activation assay kits (Cytoskeleton, Inc., Denver, CO, USA), according to the manufacturer's protocols. The CECs were cultured in conditions identical to those previously described in the cell migration assay. The cells were washed with ice-cold PBS and lysed in ice-cold lysis buffer with the addition of a 1:100 final dilution of a protease inhibitor cocktail (Cytoskeleton, Inc., Denver, CO, USA). Lysed cells were harvested with a cell scraper and centrifuged at $10,000 \times \mathrm{g}$ at $4^{\circ} \mathrm{C}$ for $1 \mathrm{~min}$. The supernatant was aliquoted, snap frozen in liquid nitrogen and stored at $-70^{\circ} \mathrm{C}$ until further use. An equal volume of supernatant was incubated for $1 \mathrm{~h}$ at $4^{\circ} \mathrm{C}$ in the presence of glutathione S-transferase fused p21 activated kinase 1-p21 binding domain
A

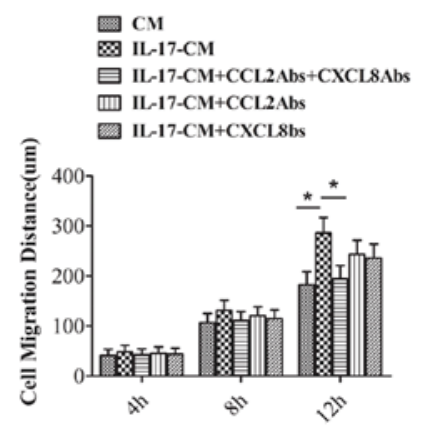

B

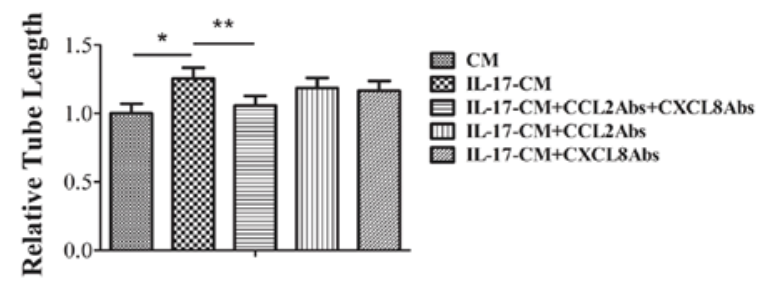

Figure 3. IL-17-CM promotes CEC migration and tube formation via CXCL8 and CCL2. (A) CEC monolayers were injured and subsequently incubated with CM, IL-17-CM with or without neutralizing antibodies to CXCL8 and/or CCL2. Wound healing distance was recorded by phase contrast microscopy over a $12 \mathrm{~h}$ time course following the scratch injury. (B) CECs were seeded onto Matrigel-coated plates and exposed to CM, IL-17-CM with or without neutralizing antibodies to CXCL8 and/or CCL2 for $16 \mathrm{~h}$. Endothelial tube formation was visualized by microscopy and the tube length was analyzed. Values are presented as the mean \pm standard deviation of three replicate experiments in A and five in $\mathrm{B} .{ }^{*} \mathrm{P}<0.05 ;{ }^{* *} \mathrm{P}<0.01$. IL-17, interleukin-17; $\mathrm{CM}$, conditioned medium; CCL2, C-C motif chemokine ligand 2; CXCL8, C-X-C motif chemokine ligand 8; CEC, choroidal endothelial cell; Abs, neutralizing antibody.

beads for GTP-Rac1 adsorption, or Rhotekin-Rho binding domain bound to glutathione-sepharose beads for GTP-Rho adsorption. The supernatant was subsequently washed three 


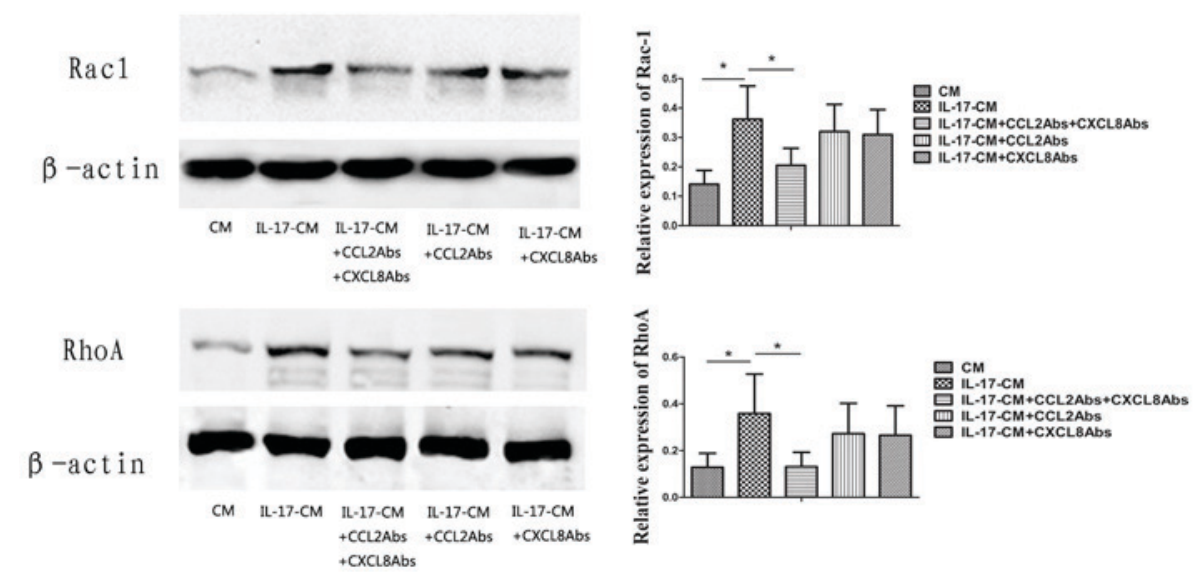

Figure 4. IL-17-CM increases Rac1 and RhoA activity via CXCL8 and CCL2. Activation of (A) Rac1 and (B) RhoA via CXCL8 and CCL2 in IL-17-CM. Cells were incubated with CM or IL-17-CM with or without neutralizing antibodies to CXCL8 and/or CCL2 for 10 min, and were subsequently subjected to Rac1 and RhoA pull-down assays and western blot analysis. Data is presented as the mean \pm standard deviation of results from at least three independent experiments. ${ }^{*} \mathrm{P}<0.05$. Rac1, Ras-related C3 botulinum toxin substrate 1; RhoA, Ras homolog gene family, member A; IL-17, interleukin-17; CM, conditioned medium; CCL2, C-C motif chemokine ligand 2; CXCL8, C-X-C motif chemokine ligand 8; Abs, neutralizing antibody.

times with the wash buffer. Bound proteins were eluted in sample buffer and analyzed by western blot analysis using antibodies against Rac1 and RhoA. All materials used including those for the western blot analysis, were included in the pull-down assay kits.

Statistical analysis. Data are presented as the mean \pm standard deviation, using SPSS software v11.0 (SPSS, Chicago, IL, USA) the cell proliferation assay and ELISA results between groups were statistically compared using the Student's t-test. Tube formation assay and migration assays results between CM and IL-17-CM were analyzed using the Student's t-test. Tube formation, migration and the Racl and RhoA pull-down assays were analyzed using one-way analysis of variance, followed by a Bonferroni correction test as a post-hoc test. $\mathrm{P}<0.05$ was considered to indicate a statistically significant difference.

\section{Results}

IL-17-CM enhances migration and tube formation, however not proliferation of CECs. CM and IL-17-CM induced the proliferation of CECs, however there was no significant difference in the relative absorbance of the supernatant between the two groups at 24, 48 and $72 \mathrm{~h}$ (Fig. 1A). IL-17-CM significantly increased tube formation in CECs. The data was normalized to a $\mathrm{CM}$ value of 1 , with the relative tube length of CECs stimulated by IL-17-CM at $1.25 \pm 0.08$ ( $\mathrm{P}=0.001$; Fig. 1B). The migration distance of the CECs increased with duration in both the IL-17-CM and the CM treated groups. The migration distance of CECs stimulated with IL-17-CM was significantly increased at $12 \mathrm{~h}$ in comparison with $\mathrm{CM}$ (Fig. 1C; CM, $185 \pm 26 \mu \mathrm{m}$; IL-17-CM, 285 $\pm 30 \mu \mathrm{m} ; \mathrm{P}=0.012$ ).

IL-17 fails to upregulate VEGF secretion by the ARPE-19 monolayer. To investigate whether migration and tube formation of CECs was induced by VEGF secreted by the RPE, the expression of VEGF was detected in the supernatant of the ARPE-19 monolayer with or without IL-17 stimulation.
The expression levels of VEGF in the supernatant of ARPE-19 with or without IL-17 stimulation were not significantly different (Fig. 2; IL-17-CM, 0.19 $\pm 0.12 \mathrm{pg} / \mu \mathrm{g}$ total protein; $\mathrm{CM}, 0.16 \pm 0.09 \mathrm{pg} / \mu \mathrm{g}$ total protein).

IL-17-CM promotes the migration and tube formation of CECs via CXCL8 and CCL2. In the author's previous study, the levels of CXCL8 and CCL2 increased markedly in the supernatant from RPE cells treated with IL-17 (16). To investigate the potential role of CXCL8 and CCL2 in the migration of CECs in IL-17-CM, CXCL8 and/or CCL2 expression was neutralized in IL-17-CM prior to migration and tube formation assays. The migration distance and tube formation ability of CECs in CM, IL-17-CM, IL-17-CM with anti-CXCL8 and anti-CCL2, IL-17-CM with anti-CCL2 only or IL-17-CM withanti-CXCL8 only was investigated (Fig. 3). The data was normalized to a $\mathrm{CM}$ value of 1.CEC migration was significantly inhibited by IL-17-CM with the combination ofanti-CXCL8 and anti-CCL2 ( $\mathrm{P}=0.024)$, however the inhibitory effect was not present in treatment with either antibody alone (Fig. 3A). Similarly, the addition of bothanti-CXCL8 and anti-CCL2 in IL-17-CM significantly reduced tube length compared with IL-17-CM (IL-17-CM, 1.25 \pm 0.08 ; IL-17-CM + anti-CCL2 + anti-CXCL8, 1.06 \pm 0.07 ; $=0.04)$, however addition of either antibody alone had no significant effect on tube length (Fig. 3B). The values were normalized to a $\mathrm{CM}$ value of 1 . This data suggested that the combination of CXCL8 and CCL2 expression in IL-17-CM was essential to induce the migration and tube formation of CECs.

IL-17-CM upregulates Racl and RhoA activity in CECs via CXCL8 and CCL2. The expression levels of Racl and RhoA in CECs was significantly increased in IL-17-CM treated groups in comparison with the $\mathrm{CM}$ group (Fig. 4; Rac1, $\mathrm{P}=0.008$; RhoA, $\mathrm{P}=0.042$ ). Neutralizing antibodies to both CXCL8 and CCL2 significantly inhibited Rac1 and RhoA activity in the IL-17-CM group following $10 \mathrm{~min}$ of treatment (Rac1, $\mathrm{P}=0.037$; RhoA, $\mathrm{P}=0.038)$. A significant inhibition of $\mathrm{Rac1}$ and RhoA activity was not present when CXCL8 or CCL2 alone 
were neutralized with the respective antibodies. Collectively, these results suggested that IL-17-CM induced the upregulation of Rac1 and RhoA activity in CECs, which may be in part mediated by CXCL8 and CCL2.

\section{Discussion}

The present study demonstrated that a potent angiogenic effect was induced in human CECs treated with IL-17-CM from RPE cells, via the promotion of migration and capillary tube formation, however not proliferation. The IL-17-CM-induced angiogenic response was largely mediated by CCL2 and CXCL8. VEGF expression levels were not affected by the IL-17-CM.

To physiologically mimic RPE cells in vivo, ARPE-19 monolayer was cultured for 8 weeks on Transwell filter, which may mimic differentiation to some degree. No significant difference was observed between CM and IL-17-CM treated groups in the proliferation assay, suggesting that IL-17 failed to induce mitogenic activity in RPE. However, it was demonstrated that IL-17-CM significantly increased the migration and capillary tube formation of CECs in comparison with CM from unstimulated RPE. A wound healing assay was performed to detect cell migration (19). Results suggested that the wound closure was a result of both cell migration and proliferation. Proliferation cannot be completely excluded from the involvement of wound closure in the present study, as there was no significant difference in proliferation between the CM and IL-17-CM-treated groups. These results suggest that cell migration may be a more important contributor to wound healing. Therefore, other angiogenic factors in IL-17-CM were further explored and the expression levels of VEGF were detected. A previous study demonstrated that IL-17 may induce angiogenesis through the upregulation of VEGF expression (20). The results of the present study indicated that RPE in vitro was capable of secreting VEGF, however IL-17 had no significant effect on VEGF expression. In the aforementioned previous study, a different cell type was used to investigate VEGF expression, which may explain the contradicting results. In the present study, the increase in migration and capillary tube formation ability in CECs induced by IL-17-CM was independent of VEGF. Therefore, the angiogenic effect of IL-17-CM may be mediated by factors other than VEGF.

The author's previous study suggested that CCL2 and CXCL8 expressionis significantly increased in RPE stimulated by IL-17 (16). CCL2 and CXCL8 are angiogenic, promoting the migration and capillary tube formation of endothelial cells (21). In the present study, no anti-angiogenic effect was observed through the addition of anti-CCL2 or anti-CXCL8 antibodies in IL-17-CM. However, the migration and capillary tube formation ability of CECs was significantly decreased in the presence of anti-CCL2 and anti-CXCL8 antibodies combined. Therefore, CCL2 and CXCL8 may be key pro-angiogenic factors in IL-17-CM, however CCL2 or CXCL8 alone do not induce a distinct pro-angiogenic effect.

The migration of CECs towards the RPE and neurosensory retina is a key feature $\mathrm{f} \mathrm{CNV} \mathrm{development.} \mathrm{Filopodia,}$ lamellipodia and stress fibers may promote endothelial cell migration via actin rearrangement, through the activation of biochemical pathways that are primarily regulated by Rho
GTPases, including Racl and RhoA (22). Racl is required for lamellipodium extension, and RhoA is associated with stress fiber formation $(23,24)$. In the present study, neutralizing CCL2 and CXCL8 expression decreased Rac1 and RhoA activity in IL-17-CM, suggesting that IL-17 increased CCL2 and CXCL8 expression in RPE to promote CEC migration, which may be mediated by Rac1 and RhoA.

In conclusion, IL-17-CM was demonstrated to induce potent angiogenic activity. The suppression of CEC migration and capillary formation in IL-17-CM by anti-CCL2 and CXCL8 antibodies indicated that IL-17 may indirectly mediate the development of new choroidal vessels via the RPE in an in vitro wet AMD model. Results suggest that IL-17 may be a novel therapeutic target for the treatment of $\mathrm{CNV}$ in AMD.

\section{Acknowledgements}

The present study was supported by The National Natural Science Foundation of China (grant nos. 81200704 and 81200677), The National Basic Research Program of China (program 973, grant no. 2011CB510200) and The National Key Clinical Specialties Construction Program of China.

\section{References}

1. Lim LS, Mitchell P, Seddon JM, Holz FG and Wong TY: Age-related macular degeneration. Lancet 379: 1728-1738, 2012.

2. Van Lookeren Campagne M, LeCouter J, Yaspan BL and Ye W: Mechanisms of age-related macular degeneration and therapeutic opportunities. J Pathol 232: 151-164, 2014.

3. Grossniklaus HE and Green WR: Choroidal neovascularization. Am J Ophthalmol 137: 496-503, 2004.

4. Frank RN: Growth factors in age-related macular degeneration: Pathogenic and therapeutic implications. Ophthalmic Res 29: 341-353, 1997.

5. Mousa SA, Lorelli W and Campochiaro PA: Role of hypoxia and extracellular matrix-integrin binding in the modulation of angiogenic growth factors secretion by retinal pigmented epithelial cells. J Cell Biochem 74: 135-143, 1999.

6. Campochiaro PA: Retinal and choroidal neovascularization. J Cell Physiol 184: 301-310, 2000.

7. Holz FG, Pauleikhoff D, Klein R and Bird AC: Pathogenesis of lesions in late age-related macular disease. Am J Ophthalmol 137: 504-510, 2004.

8. Zarbin MA: Current concepts in the pathogenesis of age-related macular degeneration. Arch Ophthalmol 122: 598-614, 2004.

9. Strauss O, Heimann H, Foerster MH, Agostini H, Hansen LL and Rosenthal R: Activation of L-type $\mathrm{Ca} 2+$ channels is necessary for growth factor-dependent stimulation of VEGF secretion by RPE cells. Invest Ophthalmol Vis Sci 44: 3926, 2003.

10. Elner VM, Burnstine MA, Strieter RM, Kunkel SL and Elner SG: Cell-associated human retinal pigment epithelium interleukin- 8 and monocyte chemotactic protein-1: Immunochemical and in-situ hybridization analyses. Exp Eye Res 65: 781-789, 1997.

11. Higgins GT, Wang JH, Dockery P, Cleary PE and Redmond HP: Induction of angiogenic cytokine expression in cultured RPE by ingestion of oxidized photoreceptor outer segments. Invest Ophthalmol Vis Sci 44: 1775-1782, 2003.

12. Shin JI and Bayry J: A role for IL-17 in age-related macular degeneration. Nat Rev Immunol 13: 701, 2013.

13. Maddur MS, Miossec P, Kaveri SV and Bayry J: Th17 cells: Biology, pathogenesis of autoimmune and inflammatory diseases, and therapeutic strategies. Am J Pathol 181: 8-18, 2012.

14. Liu B, Wei L, Meyerle C, Tuo J, Sen HN, Li Z, Chakrabarty S, Agron E, Chan CC, Klein ML, Chew E, et al: Complement component C5a promotes expression of IL-22 and IL-17 from human $\mathrm{T}$ cells and its implication in age-related macular degeneration. J Transl Med 9: 1-12, 2011. 
15. Hasegawa E, Sonoda KH, Shichita T, Morita R, Sekiya T, Kimura A, Oshima Y, Takeda A, Yoshimura T, Yoshida S, et al: IL-23-independent induction of IL-17 from $\gamma \delta$ T cells and innate lymphoid cells promotes experimental intraocular neovascularization. J Immunol 190: 1778-1787, 2013.

16. Chen Y, Kijlstra A, Chen Y and Yang P: IL-17A stimulates the production of inflammatory mediators via Erk1/2, p38 MAPK, PI3K/Akt, and NF- $\kappa$ B pathways in ARPE-19 cells. Mol Vis 17: 3072-3077, 2011.

17. Chen Y, Zhong M, Liang L, Gu F and Peng H: Interleukin-17 induces angiogenesis in human choroidal endothelial cells in vitro. Invest Ophthalmol Vis Sci 55: 6968-6975, 2014.

18. Chen Y, Yang P, Li F and Kijlstra A: The effects of Th17 cytokines on the inflammatory mediator production and barrier function of ARPE-19 cells. PLoS One 6: e18139, 2011

19. Liang CC, Park AY and Guan JL: In vitro scratch assay: A convenient and inexpensive method for analysis of cell migration in vitro. Nat Protoc 2: 329-333, 2007.
20. Honorati MC, Cattini L and Facchini A: IL-17, IL-1beta and TNF-alpha stimulate VEGF production by dedifferentiated chondrocytes. Osteoarthritis Cartilage 12: 683-691, 2004.

21. Gálvez BG, Genís L, Matías-Román S, Oblander SA, Tryggvason K, Apte SS and Arroyo AG: Membrane type 1-matrix metalloproteinase is regulated by chemokines monocyte-chemoattractant protein-1/ccl2 and interleukin-8/CXCL8 in endothelial cells during angiogenesis. J Biol Chem 280: 1292-1298, 2005.

22. Lamalice L, Le Boeuf F and Huot J: Endothelial cell migration during angiogenesis. Circ Res 100: 782-794, 2007.

23. Ridley AJ and Hall A: The small GTP-binding protein rho regulates the assembly of focal adhesions and actin stress fibers in response to growth factors. Cell 70: 389-399, 1992.

24. Ridley AJ, Paterson HF, Johnston CL, Diekmann D and Hall A: The small GTP-binding protein rac regulates growth factor-induced membrane ruffling. Cell 70: 401-410, 1992. 